\title{
Géolinguistique
}

17 | 2017

Varia

\section{Variation dialectale du portugais parlé au sein de la communauté de Montréal}

Dialectal Variation of Portuguese Spoken in the Montreal Community

\section{Fabio Scetti}

\section{(2) OpenEdition}

\section{Journals}

Édition électronique

URL : http://journals.openedition.org/geolinguistique/407

DOI : $10.4000 /$ geolinguistique.407

ISSN : 2650-8176

Éditeur

UGA Éditions/Université Grenoble Alpes

\section{Édition imprimée}

Date de publication : 1 décembre 2017

Pagination : 151-175

ISBN : 978-2-37747-025-9

ISSN : 0761-9081

\section{Référence électronique}

Fabio Scetti, « Variation dialectale du portugais parlé au sein de la communauté de Montréal », Géolinguistique [En ligne], 17 | 2017, mis en ligne le 01 février 2019, consulté le 29 octobre 2020. URL http://journals.openedition.org/geolinguistique/407 ; DOI : https://doi.org/10.4000/geolinguistique. 407 


\title{
Variation dialectale du portugais parlé au sein de la communauté de Montréal
}

\author{
Fabio Scetti \\ Université Sorbonne Nouvelle - Paris 3, CLESTHIA
}

\section{Résumé}

La migration portugaise a donné à la ville de Montréal l'un des plus florissants quartiers portugais du Canada: la comunidade. Le portugais est la langue véhiculaire dans la comunidade et le drapeau de l'identité du quartier. Bien que le portugais soit promu comme langue de pouvoir au niveau international, l'analyse des pratiques orales montre sa variation dialectale. Le but de cette contribution est donc de mettre en lumière les traits phonétiques et structurels des variétés régionales portugaises du nord du Portugal, de Madère et des Açores dans ce contexte particulier. Les résultats montrent que les pratiques linguistiques à l'école mettent en valeur la norme standard. Mais l'analyse des pratiques orales souligne que l'évolution des normes se dirige vers ces variétés régionales et vers la norme du portugais brésilien.

\section{Mots-clés}

Variation dialectale, portugais, migration, Montréal, contact de langues.

\section{Abstract}

The Portuguese migration has given to the city of Montreal one of the most flourish Portuguese district of Canada. Portuguese is a vehicular language within the district and a flag of their identity. Although Portuguese is promoted as language of power at international level, oral practices analysis shows dialectal variation. The aim of this paper is to examine phonetical and structural features of regional varieties of Portuguese: 
Northern Portugal, Madeira and Azores Islands. The results show that institutionalized linguistic practices at school are important. But, analysis of oral practices underlines a tendency of evolution of the norms towards regional varieties and to Brazilian Portuguese.

\section{Keywords}

Dialectal variation, Portuguese, migration, Montreal, language contact.

\section{Préambule}

Ce travail se veut le témoin de l'intégration du contact de langues dans une étude de variation linguistique, dans un contexte bien précis qui est celui de la migration portugaise dans la ville de Montréal à partir des années 1950. Il s'agit d'une migration différente de celle d'aujourd'hui où le migrant fait un parcours «individualisé» (Deprez, 2005). Mais, en ce qui concerne la langue parlée, il s'avère que bien que «les locuteurs de même origine nationale parlent la même "langue" officiellement, ils n'en partagent pas les mêmes normes, ni les mêmes valeurs » (Deprez, 2005). La migration a changé et les langues parlées sont différentes parce qu'elles témoignent du passage du temps et parfois du changement d'espace, une déterritorialisation. Et, rares sont les études de variations au sein d'un contexte de migration où les variations dialectales n'en sont pas cachées ${ }^{1}$.

Le concept de «communauté linguistique» serait-il alors remis en question? Dans notre étude, ce concept trouve sa place dans un espace propre (au sein d'un quartier qui se définit comme «ethnique» au Canada) et dans un temps précis (la migration portugaise des années 1950 à 1990). Le quartier de Saint-Louis est ainsi le centre de la vie communautaire des descendants de la migration portugaise à Montréal. Ces membres vivent et font vivre la comunidade (communauté) par la survie de leur langue.

Les théories variationnistes portent sur des travaux de variations sur le lexique, sur la syntaxe et sur la phonétique. Dans tous les cas, l'oral des

1. Parmi ces études, nous retrouvons les contributions de Dominique Caubet (2001) sur les études sur les variations dialectales de l'arabe (Vicente, 2007; Benítez Fernández, 2017). Mais aussi d'autres études comme celles sur les variations de l'espagnol entre structure, contact et lexique (Silva-Corvalán, 1994; Zentella, 1997; Fitch, 2013), du portugais dans le contexte de migration d'Andorre (Mazzoli, 2005), sur l'arménien occidental (Donabédian, 2000), ou encore sur la variation dialectale au sein de la migration chinoise (Zheng, 1995; Tzen, 2000). 
langues standard est mis en avant. Nous voulons ici présenter cette étude qui prend en considération les origines régionales des locuteurs et des locutrices au sein de notre groupe et surtout leurs variétés de portugais. Ces variétés font partie aujourd'hui, à tous les égards, du portugais parlé dans la comunidade. Nous devons alors réinterpréter ces variations dialectales «en contact» (Trudgill, 1986) comme des marques sociolinguistiques. Après une brève présentation du contexte et de la méthodologie adaptée à notre étude, nous présentons les traits phonétiques et structurels observés et qui nous montrent la variation régionale du portugais parlé à Montréal.

\section{Le contexte de la comunidade portugaise de Montréal}

Montréal accueille la deuxième plus grande communauté de descendants de l'immigration portugaise au Canada. La comunidade (Eusébio, 2001) représente un exemple d'union et de force sous les couleurs du même drapeau portugais. Dans le quartier de Saint-Louis, la comunidade est depuis les années 1950 «l'île portugaise» de la ville québécoise; Montréal étant le rond-point de la politique d'accueil des immigrés au Canada, entre le multiculturalisme canadien et l'interculturalisme francophone québécois. Cette présentation du contexte nous aide à comprendre, outre l'histoire de l'installation du groupe, l'importance du portugais comme langue véhiculaire de la vie du groupe au sein d'un contexte de contact de langues dominé par deux langues dominantes : le français et l'anglais.

\subsection{La diaspora portugaise dans le monde}

Le Portugal a construit son histoire, son autonomie et son indépendance, grâce aux grands voyages. À partir de $1415^{2}$, le Portugal a construit un des empires les plus grands et les plus puissants de l'histoire et le premier empire mondial réparti sur les cinq continents, qui correspond aujourd'hui à 53 États souverains différents. Ce fut le plus long des empires coloniaux européens, qui pris fin officiellement en 2002, date de l'indépendance du Timor oriental.

Durant la période de domination et de contrôle du Portugal sur ses colonies, de nombreux Portugais se sont installés dans des territoires, dans des conditions différentes, souvent de façon temporaire à des fins commerciales. À partir du XIX ${ }^{\mathrm{e}}$ siècle et au début du $\mathrm{XX}^{\mathrm{e}}$ siècle, l'installation des

2. Par convention, 1415 désigne le début de la conquête portugaise, avec la conquête de la ville de Ceuta, actuellement enclave espagnole au Maroc. 
colons portugais et d'autres européens s'est accélérée grâce aux moyens de transport maritimes (bateaux à vapeur, transatlantiques), en particulier vers les Amériques, c'est ce qu'on appelle la «grande émigration européenne».

L'émigration portugaise, diáspora portuguesa ${ }^{3}$, qui privilégiait les territoires coloniaux dont le riche Brésil, devenu indépendant en $1822^{4}$, prit de l'importance à l'époque du régime dictatorial d'António de Oliveira Sa$\operatorname{lazar}^{5}$ (1933-1968), dont l'apogée aura lieu après la révolution des Eillets ${ }^{6}$ en avril 1974. Cet exode motivé par une ascension socio-économique, qui dans un premier temps avait été contrôlé et canalisé par le régime autoritaire et conservateur vers les colonies, s'orienta alors vers d'autres pays de l'Amérique, en particulier le Venezuela et l'Uruguay au sud, le Canada et les États-Unis au nord. Cependant, durant les années 1970 et 1980, le Portugal subit des changements importants qui transformèrent complétement le pays. De dictature et de grand empire colonial, en l'espace de dix ans, le Portugal est devenu membre de la $\mathrm{CEE}^{7}$, le $1^{\text {er }}$ janvier $1986^{8}$. Par la suite, les flux migratoires se dirigèrent vers les pays européens voisins.

De nos jours, nous pouvons encore observer la présence de groupes issus de ces flux migratoires dans plusieurs grandes villes au monde. Au Canada, l'émigration portugaise a laissé son empreinte dans les provinces de l'Ontario, dans le Manitoba au centre, dans la Colombie-Britannique et au Québec, dont la plus grande communauté est celle de Montréal.

3. Diaspora : concept assez vaste qui est ici utilisé en portugais où son emploi est plus fréquent. Il existe un Conseil de la diaspora portugaise, qui a été créé en 2012 avec le patronage du président de la République portugaise : <www.diasporaportuguesa. org $>$.

4. 7 Septembre 1822: O grito de Ipiranga (indépendance ou mort) date de commémoration de l'indépendance de l'empire du Brésil.

5. António de Oliveira Salazar - homme politique portugais, à la base professeur d'économie à l'université de Coimbra, président du Conseil des ministres de 1932 à 1968 et fondateur du régime autoritaire dictatorial de l'Estado Novo (État nouveau).

6. Révolution des EEillets - Revolução dos Cravos ou 25 de Abril (25 avril 1974), date qui marque le coup d'État militaire et la chute du régime autoritaire de l'Estado Novo (État nouveau), instauré par António de Oliveira Salazar en 1933.

7. CEE - Communauté économique européenne, jusqu'en 1985 composée par 10 membres : les 6 fondateurs - France, Italie, Pays-Bas, Allemagne, Belgique et Luxembourg (1958) - , Danemark, Irlande et Royaume-Uni d'un premier élargissement (1973) et la Grèce après le deuxième élargissement (1981).

8. $1^{\text {er }}$ janvier 1986 - signature de l'adhésion officielle du Portugal et de l'Espagne au sein de la CEE. 


\subsection{L'immigration portugaise à Montréal}

Les contacts entre le Portugal et le Canada ont débuté à la fin du Xve siècle, quand des pionniers portugais cherchant une route vers les Indes se sont intéressés au Canada oriental. Néanmoins, les relations entre les deux pays, à l'époque contemporaine, s'intensifient à partir des années 1950, par un mouvement migratoire soutenu des Portugais vers ce pays.

En fait, les deux pays ont alors commencé à construire un projet d'immigration contrôlée. En 1951, la Junta da Emigração ${ }^{9}$, qui contrôlait les flux migratoires portugais vers l'extérieur, a contacté le consul portugais à Montréal pour définir un projet d'immigration stable (Almeida Moura \& Soares, 2003). Et, afin de soulager la surpopulation dans les archipels de Madère et des Açores, le Canada était alors disposé à recevoir un groupe pilote en $1953^{10}$.

Dans les mois et les années qui ont suivi cette date, sont arrivés d'autres bateaux ayant pour destination le Canada, et les demandes de départ ayant augmenté, la commission de sélection canadienne, basée à Paris, se déplaçait au Portugal pour des entretiens rigoureux suivis d'examens médicaux des candidats potentiels. À partir de 1954, de nombreux Portugais, en majorité Açoriens, sont accueillis au Canada et constituent déjà une véritable «communauté» portugaise dans ce pays.

Dorénavant, les Portugais et les descendants de cette immigration, majoritairement originaires des îles (Açores et Madère) et du Portugal du Nord et du Centre, sont organisés en communautés que l'on retrouve dans tout le Canada : Toronto, Montréal, Vancouver, Hamilton, Ottawa-Gatineau, London (Ontario) et Winnipeg. C'est au sein de ces quartiers communautaires que se créent les premiers contacts entre de nombreux continentaux et des îliens. À présent, Montréal est la seule ville où l'on retrouve un équilibre entre les continentais (continentaux) et ceux des îles, en particulier les Açoriens.

Aujourd'hui, la «communauté portugaise» de Montréal compte un total d'environ 40000 individus selon l'origine ethnique (Statistiques Canada, 2003), 30000 selon la langue maternelle, d'après les dernières statistiques nationales (Statistiques Canada, 2009). Montréal comporte la concentration de Portugais et de descendants de l'immigration portugaise la plus

9. Junta da Emigração (Conseil de l'émigration) - organe d'État, crée en 1947 sous la dictature de Salazar et qui contrôlait les flux migratoires à partir du Portugal.

10. Les premiers immigrés réguliers, de l'immigration officielle et encadrée, sont arrivés au Canada, plus précisément à Halifax en Nouvelle-Écosse, en 1953 sur le bateau italien Saturnia. 
importante de la province du Québec et la deuxième du Canada, après Toronto et juste avant Vancouver. Aujourd'hui, la «communauté » de Montréal, s'étend depuis la ville jusque dans sa banlieue.

Le plan du Grand Montréal (région métropolitaine), ci-dessous (fig. 1), illustre la distribution et la concentration de la population portugaise selon la citoyenneté et le lieu de naissance. Les deux indicateurs croisés montrent :

- en orange une fréquence entre 110 et 350 individus ( 24 communes);

- en jaune une fréquence entre 40 et 110 individus (111 communes);

- en blanc une fréquence entre 10 et 40 individus (229 communes);

- en bleu clair une fréquence entre 0 et 10 individus (147 communes);

- en bleu foncé une fréquence de 0 individu.

La population portugaise se concentre au sein de la comunidade (quartier des Portugais, à Saint-Louis), dans des communes à l'est et au nord de la ville et dans des communes de la banlieue, à Laval et de l'autre côté de la rivière Saint-Laurent.

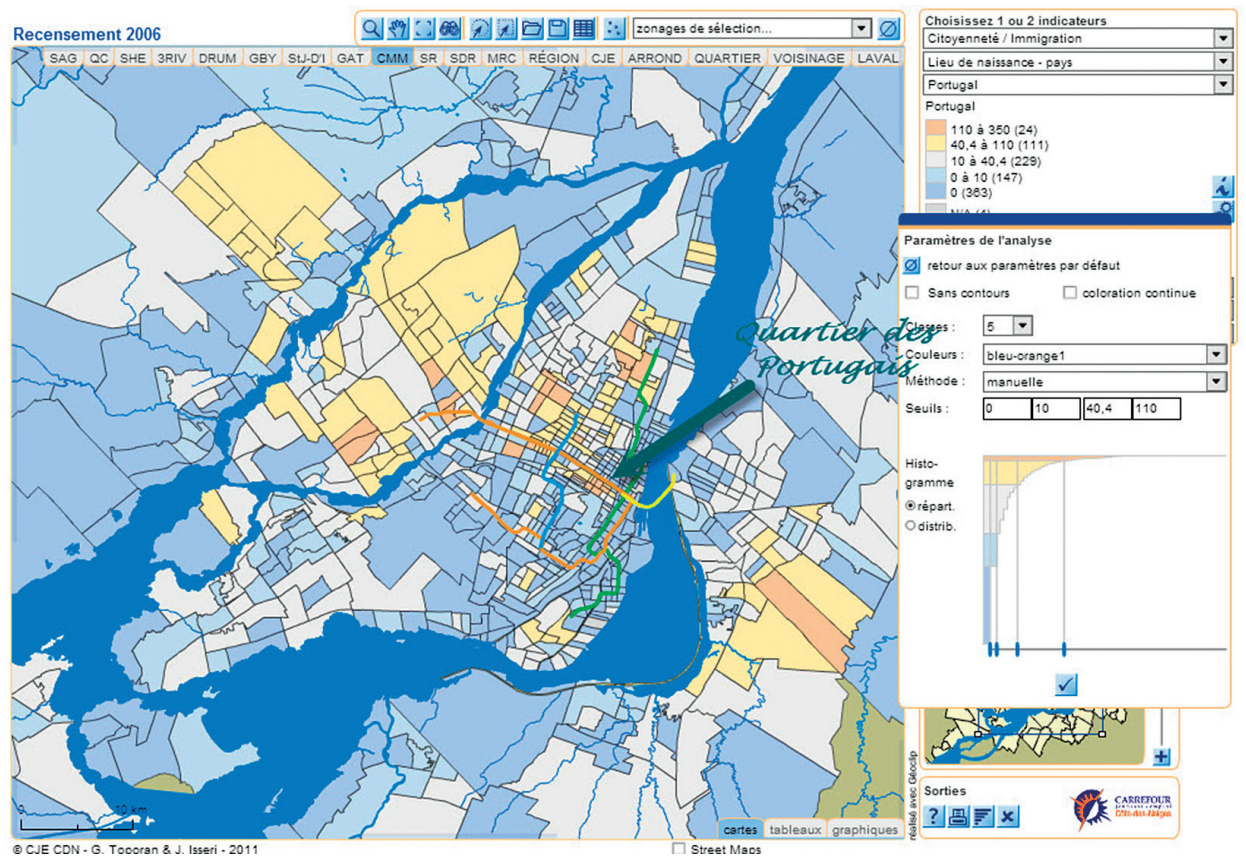

Figure 1. - Recensement 2006 (Statistiques Canada, 2009).

Citoyenneté et lieu de naissance : Portugal. 


\subsection{La création du quartier portugais à Montréal}

Les premiers Portugais arrivés à Montréal se sont installés dans le quartier de Saint-Louis, en centre-ville. Ce quartier, aujourd'hui centre culturel et rendez-vous des Portugais de la ville de Montréal, du Grand Montréal (région métropolitaine) et de toute la province du Québec, fait partie de l'arrondissement du Plateau-Mont-Royal.

L'installation définitive du groupe à Saint-Louis a été marquée par la construction de maisons et le développement d'une vie commerciale active où la langue portugaise est l'une des langues d'échanges. Nous pouvons y trouver des restaurants, des boutiques, des bars et des clubs des différentes régions lusitaines.

Récemment, beaucoup de familles d'origine portugaise se sont délocalisées vers les banlieues de la ville (Da Rosa \& Teixeira, 2000), mais maintiennent dans Saint-Louis leur centre de référence et leur point de rencontre. Le portugais se maintient ici comme la langue du groupe qui est véhiculée au sein des institutions et grâce à l'apport des médias.

De plus, la langue portugaise est devenue une clé d'intégration pour la récente immigration brésilienne qui, n'ayant pas de communauté établie et un espace bien délimité comme celui des Portugais, s'appuie sur les institutions portugaises (Almeida, 2014). À l'école, la norme privilégiée est le portugais européen (PE) ou norma-padrão, comme seule option d'enseignement. À la télévision, par contre, le PE souffre de la force médiatique du portugais brésilien $(\mathrm{PB})$ qui a saturé les maisons grâce à ses séries et telenovelas, même au Portugal. Cette lutte entre PE et PB sur le plan international décrit le portugais comme véritable exemple de «langue pluricentrique» (Clyne, 1992; Muhr, 2012), où plusieurs centres sollicitent la primauté. Dans cette recherche, il est alors inévitable de considérer l'importance de la stricte relation qui se crée entre langue et pouvoir, dans le contexte de cette «lutte des normes».

\subsubsection{La norma-padrão seule langue à l'école communautaire}

Au sein de la comunidade, l'école, dite «communautaire», joue un rôle de rééquilibrage en faveur du $\mathrm{PE}$, ce qui peut créer un phénomène de spectacularisation par rapport à la pratique d'autres variétés, et notamment celles des îles, considérées comme des «dialectes» très éloignés de la norme de prestige et qui souffrent d'une stigmatisation.

L'école devient une institution de véritable importance pour le maintien de la langue dans un contexte d'immigration, où elle se retrouve minoritaire, mais elle joue un rôle important de «compensatrice» là où il y a des limites d'exposition à la langue et de pratique de la part des locuteurs et locutrices, en excluant les variations régionales. 
La norma-padrão, basée sur la variante du PE central (ligne qui va de Lisbonne à Coimbra) est aussi promue dans le monde entier par l'Institut Camões ${ }^{11}$, et enseignée comme le "véritable portugais».

Dans l'école de la Missão Santa Cruz (MSC) de Montréal, supportée par le Camões, elle est enseignée comme la «bonne» et l'école prend alors la position de seul référent. Il est intéressant d'observer qu'au sein de l'école de la MSC les enseignants sont souvent originaires du Portugal du centre, porteurs alors de la pratique de cette variété reconnue comme normative, la «langue de référence» (Dabène, 1994 : 21). Cette décision pourrait expliquer la faible présence d'élèves originaires des îles, dont les Açoriens qui représentent un pourcentage important au sein des descendants de la migration portugaise de Montréal (Eusébio, 2001).

\subsubsection{Le portugais à la télévision}

Le rôle de la télévision a été fondamental, à partir des années 1950 et 1960 , comme promotrice de la «bonne» langue sur le plan national. Au Portugal aussi, la télévision est devenue éducatrice permettant un recul important du taux d'analphabétisme qui en 1960 touchait 40,3\% de sa population (Nobre Correia, 1997). La télévision et notamment la chaîne nationale RTP (Rádio e Televisão de Portugal ${ }^{12}$ ) et sa version internationale RTPi, pour les Portugais dans le monde, sont devenues alors, de véritables «machines d'éducation».

À partir de ces années, les médias de la diáspora et en particulier la télévision, ont réussi à réduire la distance géographique entre le Portugal et le pays d'immigration, et ont eu une influence non négligeable dans l'espace public sur la construction d'une identité portugaise à l'étranger. Grâce à la langue, les discours médiatiques ont aidé les Portugais dans le monde, à rester à jour avec le quotidien du pays, et ont facilité la reconfiguration d'un esprit de nation unie et la construction d'un transnationalisme portugais, basé sur l'histoire commune et le lien avec le territoire.

Selon Benedict Anderson (1992), les médias sont importants pour définir le «nationalisme à distance». Ce qui a été le cas du Portugal, qui

11. Camões - Instituto da Cooperação e da Língua (Institut de la Coopération et de la Langue) - Institut publique qui a succédé en 1992 à l'Instituto da Cultura e Língua Portuguesa (Institut de la Culture et de la Langue portugaise). Il est présent dans 30 pays où il a créé des Centros de Língua Portuguesa dans le but de promouvoir la langue et la culture portugaises à l'étranger ( $<w w w . i n s t i t u t o-c a m o e s . p t>)$.

12. Radio et Télévision du Portugal (RTP) - service public de radio et télévision au Portugal, créé en 1955. En 1972, création de RTP Madeira, en 1975 de RTP Açores et puis dans les années 1990 ouverture plus globale avec la création de RTPi (1992) et RTP África (1998). 
après la période de la décolonisation, a reconstruit une identité nationale autour de discours d'appartenance, de continuité et d'authenticité du peuple portugais.

Dans le contexte de Montréal, où les «langues d'origine» ou «langues d'héritage» ont acquis leur espace, la définition et la dénomination de «chaîne ethnique» obligent légalement les chaînes lusophones locales à transmettre $80 \%$ de leur programmation en portugais. De plus, un forfait des chaînes lusophones, un «pack national» est envisageable avec, outre les chaînes nationales du Portugal ${ }^{13}$, la chaîne brésilienne Rede Globo.

Cette situation a été favorable au maintien de la langue dans les foyers, mais la présence de cette chaîne brésilienne nous fait réfléchir sur l'importance de la langue dans les médias, et en particulier à l'importance du choix de la variante de référence, ce qui nous prépare à la lutte sous-jacente entre PE et PB dans le monde lusophone des communications.

\section{Méthodologie adaptée à l'étude}

L'étude d'une variété (Chambers, Trudgill \& Schilling-Estes, 2002) de langue dans le contexte de migration se fonde sur des considérations portant sur des situations de bilinguisme ou trilinguisme et des observations des phénomènes issus du contact entre les langues.

En tenant compte des choix méthodologiques, le fait que la langue utilisée lors des questionnaires et du premier contact avec les interviewés était le portugais, nous allons ici présenter l'enquête et l'analyse sociolinguistique des variations régionales du portugais parlé à Montréal, sans mettre en lumière les phénomènes de contact, résultats de la pratique quotidienne en mélangeant le portugais au français et/ou à l'anglais (Scetti, 2016).

\subsection{Enquête qualitative et ethnographique}

Cette contribution s'appuie sur une enquête réalisée pour la préparation d'une thèse doctorale soutenue en avril 2016 et qui a pour titre : Évolution de la langue portugaise dans sa dynamique de transmission au sein de la «communauté portugaise» de Montréal (Scetti, 2016).

La méthodologie à la base de ce projet est ethnographique et qualitative. Ethnographique, parce qu'il s'agit d'un processus en évolution (Erickson, 1984; Hymes, 1996), caractérisé par une description fine du contexte d'étude et notamment en impliquant les locuteurs et locutrices, sujets de cette étude multisituée (personnes, institutions, associations et

13. RTPi, SIC Internacional, Sport Direct. 
clubs collectifs). Qualitative, parce que le point de force est l'individu; individu comme ensemble, image ou exemple, peut être entendu aussi comme chacun-e des locuteurs et locutrices sélectionné-e-s tout au long de la recherche.

La recherche a été réalisée entre 2011 et 2014, en deux terrains. Pendant la première partie du terrain de recherche en 2011, un questionnaire a permis de mieux connaître le groupe d'étude et surtout de définir son contexte. Cet outil a été important lors de la préparation des entretiens et au moment de la sélection des interviewés. L'entretien a été l'élément de base de la recherche afin de recueillir des données en portugais oral, par différents locuteurs et locutrices au sein du groupe, le but étant de rassembler un corpus ayant un intérêt sociolinguistique et ethnographique qui pouvait être observé, commenté et ensuite expliqué. Nous avons réalisé 52 entretiens individuels et collectifs sous forme d'enregistrements (audio et vidéo). Finalement, grâce à l'observation et la prise de notes, le chercheur a pu participer aux activités et à la vie communautaire, en observant, en particulier, les pratiques orales dans des situations différentes.

\section{2. Étude sur l'évolution des pratiques en langue portugaise au sein du groupe}

Cette recherche nous a permis d'observer le parcours de Cosmetic Attrition (Scaglione, 2000) : l'érosion de la structure du portugais parlé au sein de la comunidade à Montréal.

Nous avons conduit deux analyses qui se complétaient entre elles. D'une part, l'analyse linguistique nous a permis de mettre en lumière sept points de fragilité dans les pratiques quotidiennes du portugais au sein du groupe. Nous avons remarqué le changement d'enclise à proclise des pronoms clitiques dans les verbes pronominaux, la fragilité des marques grammaticales de genre et de nombre, la fréquence d'utilisation de l'expression a gente (qui signifie «les gens» et se conjugue à la $3 \mathrm{SG}$ comme un «on» inclusif en français) et son usage comme substantif, la fragilité de la marque du subjonctif et finalement, les usages des verbes auxiliaires : estar et ser (dans la temporalité) et le déclin de l'utilisation du verbe haver dans sa fonction d'existentiel en faveur du verbe ter (Scetti, 2016).

D'autre part, cette analyse a été complétée par une analyse du discours épilinguistique (Canut, 2000). Ce croisement nous a permis d'observer comment les questions de la référence et de la norme de la langue s'imposent au sein des discours circulant au sein du groupe, et comment ces discours influencent les locutrices et locuteurs dans leur parcours de (re) construction de l'identité au sein de la comunidade (Scetti, 2016). 


\section{Le portugais parlé dans la comunidade}

Le portugais est considéré comme langue allophone dans le contexte montréalais. Il trouve sa place et son espace de pratique dans «son» quartier, au sein d'un panorama linguistique montréalais dominé par le français et l'anglais.

Après avoir réfléchi sur les références et le prestige du portugais à Montréal, notamment au sein des institutions (école et médias), nous voulons ici présenter une liste des traits phonétiques et structurels, résultats de l'étude sur la variation dialectale. Il est important de réfléchir sur l'apport linguistique dans le contexte de la migration. Joshua A. Fishman (1971), dans ses définitions de la «langue», nous propose une définition géographique de la langue en «dialectes», ce qui nous aide dans ce parcours d'explication de la déterritorialisation d'une langue et de son évolution dans un nouveau territoire. Dans son parcours de «dialectisation», évolution dans un nouveau territoire, le «portugais de Montréal», terminologie approximative par rapport au contexte, trouve ses références dans ses origines : la nature malléable et mutable de la langue dans ses variantes régionales et la fixité de la langue normative dans sa référence, la langue «standard» (Mackey, 1989).

L'importance de la langue normative et des vernaculaires des premiers arrivés nous permet de réfléchir sur l'influence de l'apport linguistique (en anglais : linguistic input). L'apport linguistique se définit comme l'apport de l'environnement linguistique : présence de la langue dans l'interaction quotidienne en famille, les médias, la scolarisation, et tous les contacts possibles avec la langue ou les langues (orale et écrite). Tous ces facteurs jouent un rôle très important dans le processus d'évolution d'une langue et dans sa transmission. Dans le contexte particulier de la migration, bien que la langue transmise soit présente au sein de l'interaction familiale ou qu'elle en soit même la seule protagoniste, son apport oral, lié aux contextes situationnels, peut ne pas être suffisant pour que les locuteurs et locutrices puissent acquérir complétement tous les aspects grammaticaux de cette langue à un niveau normatif (Montrul, 2010). La situation de contact modifie les relations sociales et les contextes de pratique, et au sein de notre étude, si dans un premier temps le portugais couvrait tous les espaces et usages, à présent il partage ses espaces de pratique avec le français et l'anglais.

Dans ce contexte, l'apport linguistique en portugais peut provenir de sources diverses, il peut se différencier selon les générations et dépendre de la pratique quotidienne de chacun-e des locuteurs et locutrices. Pour un locuteur ou une locutrice de première génération $(1 \mathrm{G})$, cet apport présent 
depuis la naissance se maintient tout au long de leur vie, en changeant tout de même. Pour les générations suivantes, l'apport linguistique en portugais est moins soutenu et provient plutôt de l'espace familial (contact avec les grands-parents, réunion en famille, voyage au pays d'origine) et à travers les médias et l'école où la langue normative enseignée serait plus présente (école communautaire en langue portugaise).

Dans notre étude, pour les locuteurs et locutrices de deuxième et troisième générations $(2 \mathrm{G}$ et $3 \mathrm{G})$, heritage speakers (locuteurs d'héritage ou locuteurs héréditaires) (Montrul, 2012), intégrées et scolarisées dans une des langues dominantes, le contact avec un apport linguistique continu en portugais est réduit et les espaces de pratique se resserrent. Le contact est limité, moins varié, et se concentre dans le milieu familial et communautaire (rencontres, fêtes, réunions, vacances au pays d'origine, et faible apport médiatique). La présence de la scolarisation en portugais peut être un facteur très important pour une acquisition plus complète des aspects grammaticaux de la langue, mais parfois, la scolarisation n'est pas suffisante à cause des horaires réduits et de l'engagement insuffisant des étudiants, qui perdent l'opportunité d'acquérir toutes les compétences de la langue (Rothman, 2009 : 156).

L'apport linguistique depuis la famille trouve un rôle important en ce qui concerne le transfert d'autres pratiques (culturelles, religieuses, culinaires, traditions), où la langue en serait un moyen. Tout au long de notre étude sur la variation dialectale du portugais parlé au sein de la comunidade de Montréal, nous avons pu identifier la présence de traits qui soulignent l'existence de variantes qui comme leurs locuteurs et locutrices, ont aussi fait un voyage.

\subsection{Variations dialectales du portugais européen}

L'observation de la variation dialectale du portugais parlé au sein de la comunidade nous a permis de travailler sur la variation du portugais européen (PE) en englobant les variations dialectales des îles, le portugais insulaire (PI) de Madère et des Açores, deux variantes distinctes et éloignées mais qui ont des similitudes (Segura \& Saramago, 1999).

Lors de l'analyse, nous avons pu souligner, tout d'abord, que dans ce contexte de migration, la présence de traits de variation dialectale est plus accentuée chez les locuteurs et locutrices de 1G, qui ont quitté le Portugal avant 1974, période où le système politique dictatorial ne privilégiait pas l'alphabétisation et l'éducation de ses citoyens.

Les variations dialectales ont pu s'exporter grâce à l'émigration du $\mathrm{xx}^{\mathrm{e}}$ siècle vers l'Europe et les Amériques. C'est seulement après la chute de la 
dictature qu'au Portugal nous avons pu assister à un processus de convergence de la langue portugaise orale vers un «modèle standardisé» basé sur la variante lisboète (Deprez, 2013). Dans les exemples ci-dessous, chaque trait de variation fait référence à cette variante, la norma-padrão du PE.

\subsubsection{Traits phonétiques du portugais parlé à Montréal}

Dans les traits phonétiques plus observés lors de notre recherche, nous retrouvons des traits distinctifs des dialectes du nord du Portugal (dialectos setentrionais) et due à une importante immigration depuis les archipels des Açores et de Madère, des traits distinctifs de ces deux régions autonomes du pays (dialectos insulares) ou PI (Mateus, 2005).

Nous présentons ci-dessous une liste de ces traits avec traduction en note, nom du locuteur ou de la locutrice, génération et provenance.

\section{a. Les dialectes du Nord}

Nous avons identifié ci-dessous, certains traits phonétiques qui caractérisent les dialectes septentrionaux et que l'on retrouve à Montréal, comme le maintien des fricatives apico-alvéolaires $/ \mathrm{s} /$ et $/ \mathrm{z} /$ et par la conservation de la diphtongue /ow/ comme dans l'exemple suivant :

(1)

Sílvia (2G - Nord) : Tenho outras [owtra]] pessoas conhecidas da família ${ }^{14}$.

De plus, nous avons souvent remarqué la disparition de l'opposition entre la consonne fricative labiodentale voisée / $\mathrm{v}$ / et la consonne occlusive bilabiale voisée /b/ qui est soulignée comme une marque identitaire de cette région au Portugal. Ensuite, nous avons souligné un emploi imprécis des formes verbales du passé simple commun des verbes ser (être) et ir (aller) - cette indifférenciation dans l'usage entre fui (1SG) et foi (3SG) peut être expliqué par la proximité des deux voyelles en question : /u/ et /o/. Dans l'exemple (2), nous pouvons observer ces deux traits :

(2)

Lurdes (1G - Nord) : Tinha vinte [bint] e dois anos foi (eu fui) pa' Manitoba ${ }^{15}$.

En revanche, il est à souligner que nous ne retrouvons pas le maintien de distinction entre l'affriquée /t $\mathrm{g} /$ (représentée graphiquement par $<\mathrm{ch}>$ ) et la fricative palatale $/ \mathrm{g} /$ (représentée graphiquement par $\langle\mathrm{x}>$ ), autre trait

14. (1) : J'ai (je connais) d'autres personnes connues par la famille.

15. (2) : J'avais 22 ans (1SG) et fut (3SG) au Manitoba. J'avais 22 ans et j'ai été au Manitoba. 
de distinction fortement souligné dans les dialectes septentrionaux, comme dans l'exemple suivant :

(3)

Esmeralda (3G - Nord) : A gente chegou [Jegow] cà no inverno ${ }^{16}$.

\section{b. Les dialectes insulaires}

En ce qui concerne la variation dialectale depuis les îles, la liste est plus étendue et plus difficile à décrire. D'une part à cause de la dissémination géographique des îles : l'archipel açoriens est formé de neuf îles et a donc de nombreuses variations dialectales au sein de la même île, et Madère trouve sa continuation dans l'île de Porto Santo. Et, d'autre part, parce que parfois un seul dialecte est pris comme référence, par exemple celui de São Miguel, le dialecte micaelense aux Açores. Ci-dessous, nous voulons présenter certains traits phonétiques observés en essayant de souligner certaines caractéristiques communes et divergentes.

Souvent, nous avons observé une instabilité de la voyelle accentuée et des altérations vocaliques qui seraient décrites comme le phénomène d'harmonisation vocalique (Segura \& Saramago, 1999). Cette harmonisation vocalique a été décrite en détail par Maria Luísa Segura et João Saramago pour l'Atlas linguístico-etnográfico de Portugal e da Galiza (ALEPG, 1999), où ils ont observé des caractéristiques communes et distinctives entre les dialectes de quatre sous-groupes en analysant aussi les caractéristiques de chacune des îles : 1'archipel de Madère (Porto Santo inclus), le groupe occidental des îles de Flores et Corvo, le groupe central de Terceira, Pico, Faial, São Jorge et Graciosa (Polášek, 2004) et le groupe oriental avec São Miguel et Santa Maria. Intéressant à observer, certaines caractéristiques sont communes entre le dialecte de l'île de Terceira et celui de Madère (Segura \& Saramago, 1999).

Il est plus difficile de parler d'un dialecte açorien que d'un dialecte madérien, vu la composition et les distances au sein de l'archipel des Açores. À Madère, par exemple, nous pouvons retrouver des éléments qui se retrouvent à Montréal parmi les locuteurs et locutrices originaires de cette île, comme la substitution de la voyelle /i/ avec le son de la semivoyelle /j/ ou la vélarisation de la voyelle tonique /a/ qui est perçue presque comme un [0]. Dans l'exemple (4), nous avons choisi une altération vocalique commune que nous avons retrouvée, celle entre $/ \mathrm{a} /$ et $/ \varepsilon /$, notamment dans certaines structures verbales comme celle de l'imparfait de l'indicatif :

16. (3) : On est arrivé ici en hiver. 
(4)

Graça (2G - Madère) : Já tinha todo pronto [...] mas eram [erem] coisas que as outras pessoas davam [dav£j] ${ }^{17}$.

Parmi les dialectes des Açores, nous avons rencontré plusieurs locuteurs et locutrices originaire de São Miguel et, lors des entretiens, nous avons pu souligner certains traits du dialecte micaelense comme l'élévation $\mathrm{du} / \mathrm{o} /$ tonique à [u] et les voyelles palatales [u] et [o] (Mateus, 2005) :

(5)

Filomena (1G-São Miguel) : Comprehende um pouco [pok] de português ${ }^{18}$.

Nous avons aussi observé la troncation de la voyelle finale /u/ écrite graphiquement $<0>$ en PE normatif. Sa réalisation se rapproche plutôt d'un son [ə] ou [i]. Cette autre altération, étudiée comme la réduction du son [u] (Cintra, 1990), peut coïncider aussi avec la troncation de la dernière syllabe ou la disparition complète de la voyelle finale. Nous pouvons remarquer, dans les exemples, que cette altération est faite par des Madériens et des Açoriens :

(6)

Teresa ( $1 \mathrm{G}$ - Madère) : Naquele tempo [tempə] não deixavam ir pa' escola francesa ${ }^{19}$.

(7)

Manuel (1G - São Miguel) : Não era o caso, naquele temp $\left[\right.$ temp ${ }^{20}$.

Deux autres éléments observés, d'un intérêt particulier, sont la palatalisation et la dépalatalisation. Le premier consiste dans la palatalisation de la consonante /1/ en / K/ (Wetzels, 2000; Matzenauer, 1999), ce qui a été déjà étudié dans des variations dialectales de Madère (8) et des Açores, spécialement à São Miguel (Andrade, 1993) (9). Le deuxième, son contraire, voit la dépalatalisation du $/ K /$, ce qui entraîne le durcissement de la diphtongue /ia/ en son [a]. Dans l'exemple (10) nous observons l'effacement de la diphtongue /ia/ et la prolongation de la voyelle [i:] antérieur au son de la consonne [1] :

17. (4) : Tout était prêt [...], il y avait des choses (vêtements) que d'autres personnes nous donnaient.

18. (5) : Il comprend un peu le français.

19. (6) : À cette époque, ils interdisaient d'aller dans les écoles françaises (francophones).

20. (7) : Ce n'était pas le cas dans cette époque-là. 
(8)

M.d.Conceição (1G - Madère) : Vim com 16 anos e comecei logo [Кogo] a trabalhar ${ }^{21}$.

(9)

Margarida (2G - São Miguel) : Só tive que começar a escola [JkoKa] em Setembro ${ }^{22}$.

Graça (2G - Madère) : Nos fins-de-semanas uniem-se na casa duma família [fami:la] outra semana na casa doutra família [fami:la] ${ }^{23}$.

Enfin, nous voulons souligner un élément qui est souvent identifié comme une marque de la variation dialectale de Madère et des Açores. Il s'agit de la centralisation de la voyelle /u/ dans un son [y]. Dans les exemples qui suivent, nous pouvons observer un locuteur de $1 \mathrm{G}$ originaire de Madère et deux originaires de São Miguel. Parmi les locutrices et locuteurs de $2 \mathrm{G}$ souvent scolarisés à l'école communautaire, nous remarquons que cette marque disparait, comme le démontre l'exemple (14) :

(11)

Agostinho (1G - Madère) : Já não tenho bem a certeza, fui [fyi] noutra fábrica ${ }^{24}$.

Manuel (1G - São Miguel) : Portugal também abriu as portas ao mundo [mynd] ${ }^{25}$.

Matilde (1G - São Miguel) : O meu marido estava na Bermuda [bermydə] ${ }^{26}$.

Elizabeth (2G-Terceira) : É muito [mujtu] importante, os meus pais sempre falaram connosco em português ${ }^{27}$.

21. (8) : Je suis arrivé à 16 ans et j'ai commencé immédiatement à travailler.

22. (9) : J'ai dû commencer l'école en septembre.

23. (10): Les fins de semaine, on se rencontrait chez une famille et la semaine suivante chez une autre famille.

24. (11) : Je n'en suis pas sûr. J'étais dans une autre usine.

25. (12) : Le Portugal a ouvert aussi les portes au monde.

26. (13) : Mon mari était aux îles Bermudes.

27. (14) : C'est très important, mes parents ont toujours parlé portugais avec nous (elle est son frère). 
Pour conclure, nous avons souligné ici certains traits phonétiques qui démarquent la variation dialectale du portugais parlé à Montréal, au sein de la comunidade. Ces éléments se définissent souvent dans les descriptions du PI (Cintra, 1971; Segura \& Saramago, 2001) ou des dialectes insulaires (Mateus, 2005), comme des éléments de différentiation et de démarcation de différence, mais ils sont aussi des marqueurs des identités régionales.

\subsubsection{Traits structurels du portugais parlé à Montréal}

Nombreux sont les traits de variation syntaxique des dialectes portugais sur tout le territoire du Portugal continental et des îles, tels que la disparition de l'article définitif devant le possessif (dans le contexte de la famille et de la parentèle) (Carrilho \& Pereira, 2011) ou les structures qui soutiennent l'usage du gérondif, par exemple : la construction du gérondif flexionné (Lobo, 2008), la séquence du verbe aspectuel (estar, ficar, andar, etc.) et la construction verbale estar + GER en substitution de la construction estar a + INF (Gonçalves, 1996; Carrilho \& Pereira, 2011). Ces éléments se retrouvent plutôt dans les variantes parlées dans la région du sud du Portugal et dans les archipels.

Nous avons décidé de mettre en lumière ici certaines structures que nous retrouvons lors de notre analyse, dont certains points qui ont été observés comme points de faiblesse du portugais parlé au sein de la comunidade (Scetti, 2016). Ces points soulignent d'une part le rapprochement entre certaines variations régionales et de l'autre un rapprochement au PB.

\section{a. Les clitiques des verbes pronominaux}

Tout d'abord, nous avons souligné le changement de position des pronoms clitiques des verbes pronominaux dans des phrases affirmatives, d'enclise (postverbale) à proclise (préverbale) (Scetti, $2016: 282$ ), phénomène bien connu dans les grammaires aussi, comme particularité du PB. Selon notre étude, ce changement de position se retrouve chez les nouvelles générations à Montréal comme le montrent les exemples suivants :

Peter (3G - Nord) : O português m'ajudou muito ${ }^{28} \rightarrow$ ajoudou-me (PE).

Filipe (3G-Centre) : Ela me diz que é mais linguísta ${ }^{29} \rightarrow$ diz-me (PE).

28. (15) : Le portugais $\mathrm{ME}+$ aida $3 \mathrm{SG}$ beaucoup. Le portugais m'a beaucoup aidé.

29. (16) : Elle $\mathrm{ME}+$ dit $3 \mathrm{SG}$ que est $3 \mathrm{SG}$ plus linguiste. Elle m'a dit qu'elle est linguiste. 


\section{b. L'usage du sujet exprimé a gente}

Ensuite, nous avons analysé l'usage du sujet a gente avec des concordances verbales différentes. Nous soulignons trois différents usages de a gente dans sa forme pronominale. Premièrement, l'option de concordance avec une forme verbale à la $3 \mathrm{SG}$ (normative), bien que très peu étudiée en PE mais soulignée comme une particularité du PB, et que nous retrouvons dans les usages de locuteurs de différentes origines au sein du groupe.

En deuxième lieu, la concordance avec une forme verbale à la 1PL. Dans les exemples suivants, nous pouvons observer l'usage de cette structure accompagnée par l'indéfini pluriel todos (tous) (17) et avec une phrase incidente (18), ce qui pourrait être analysé comme le passage de a gente à sujet explétif, sujet qui ne semble avoir aucun rôle grammatical lors de l'énoncé (Scetti, $2016: 270$ ).

Finalement, la concordance avec une forme verbale à la 3PL. Selon les études faites, cette dernière occurrence est rare dans les îles de Pico, Terceira et Graciosa (Carrilho \& Pereira, 2011), mais elle représente une caractéristique de la variante du PI de l'île de São Miguel, ce qui représente la différenciation interne avec d'autres dialectes des Açores (Segura, 2006). Cependant, nous l'avons aussi retrouvé chez des locuteurs et locutrices d'origine madérienne au sein de notre groupe à Montréal, comme le montre l'exemple (19) :

(17)

Esmeralda (2G - Nord) : A gente fomos todos obrigados ${ }^{30}$.

Manuel (1G - São Miguel) : A gente, muitas vezes digamos assim, começamos a procurar as nossas raízes ${ }^{31}$.

Iria (1G - Madère) : A gente se aprenderem e se distinguirem ${ }^{32}$.

\section{c. Le double sujet}

Un autre élément qui a été étudié avec un intérêt particulier est la structure du sujeito duplo (double sujet). Cette structure a été mise en lumière comme un trait typique de la variation madérienne (Martins, $2003: 6$ ).

30. (17) : Les gens (on) fûmes 1PL tous obligés. On a été tous obligés.

31. (18) : Les gens (on), beaucoup de fois disons 1PL ainsi, commençons 1PL à chercher nos racines. On commence à chercher nos racines, beaucoup de fois.

32. (19) : Les gens (on) SE + apprirent 1PL et SE + distinguèrent 1PL. On a appris et on s'est distingués. 
Cette structure est caractérisée par l'usage de l'expression a gente en fonction de sujet et du pronom impersonnel se qui s'ajoute au verbe à la $3 \mathrm{SG}$ en position d'enclise :

(20)

Graça ( $2 \mathrm{G}$ - Madère) : A gente era-se todos pequenos ${ }^{33}$.

L'usage du pronom impersonnel se semble être une particularité de la variation madérienne. En fait, nous retrouvons souvent son usage en position d'enclise dans des constructions avec un sujet explétif à la 1PL. Cet usage du se impersonnel semble vouloir renforcer le manque du nós (nous) comme sujet exprimé (exemple (21)). Le même se en position de proclise trouve sa place dans des constructions avec des verbes non pronominaux (exemple (19)), encore une fois avec l'usage du sujet exprimé a gente :

Graça (2G - Madère) : Se conhecemos-se ai assim ${ }^{34}$.

\section{d. Le verbe ter existentiel}

L'utilisation du verbe ter en fonction de verbe existentiel a été aussi observée. Cette construction est considérée comme une marque qui caractérise le PB par rapport au PE (Leite, Callou \& Moraes, 2003 : 101). Au Portugal, en fait, le ter existentiel a été étudié comme un phénomène de variation não padrão (non standard) du PE de la péninsule Ibérique (Carrilho \& Pereira, 2011). Mais, il ne s'agit pas d'un phénomène méconnu dans les variations insulaires et selon Aline Bazenga (2012), on parlerait d'un phénomène bien diffusé à Madère. Pendant notre recherche, nous avons pu remarquer que l'utilisation du verbe ter existentiel, au lieu du verbe haver (PE), est diffusée souvent à partir de la $2 \mathrm{G}$ et chez des locuteurs et locutrices de provenances diverses. Cela pourrait souligner l'importance de l'apport linguistique, notamment des médias, avec la force du PB comme norme privilégiée à la télévision :

(22)

Mário (2G-Centre) : Tinha días que eu ia pa' escola ${ }^{35} \rightarrow$ Havia $(\mathrm{PE})$.

33. (20) : Les gens (on) était 3SG tous jeunes. On était tous jeunes.

34. (21): $\mathrm{SE}+$ connaissons $1 \mathrm{PL}+\mathrm{SE}$ là, comme ça. Nous nous sommes connus de cette façon.

35. (22) : Il y avait des jours où j'allais à l'école. 
(23)

Sílvia (2G - Nord) : Tem muita diversidade com os tantos italianos ou os gregos $^{36} \rightarrow$ Há $(\mathrm{PE})$.

Graça (2G - Madère) : Quando chegámos aqui não tinha nada ${ }^{37} \rightarrow$ Havia $(\mathrm{PE})$.

Elizabeth (2G - Açores) : Já tinham trabalhos arranjados pela família ${ }^{38} \rightarrow$ Havia (PE).

En conclusion, dans cette partie nous avons identifié les traits structurels qui résultent de l'étude de la variation dialectale et qui sont étudiés comme des éléments de la variation du PE não padrão (non standard).

Le changement de position des clitiques, d'enclise à proclise, dans les verbes pronominaux en phrases affirmatives, l'usage du verbe ter avec une fonction d'existence, tout comme l'utilisation du sujet a gente sont des éléments étudiés comme caractérisant le PB. Nous remarquons, cependant, que ces éléments ont une pratique assez variée selon l'âge et la provenance des locuteurs et locutrices au sein de notre recherche.

L'utilisation de a gente dans sa policoncordance, en revanche, nous aide à souligner que certains usages sont limités à une région déterminée, comme l'utilisation avec la structure de double sujet à Madère (Martins, 2003).

Il devient alors compliqué d'identifier de «véritables» régions de variation syntaxique, bien que les dialectes insulaires (PI) se retrouvent souvent décrits dans la même région et se rapprochent le plus souvent des dialectes méridionaux du Portugal plutôt que des dialectes du Nord (Pereira, 2014).

\section{Sur le chemin du Brésil}

«Les gens sur le chemin du Brésil», tiré du texte de Helena Rebelo (2005), est une image non seulement représentative des individus et de leur parcours depuis le Portugal colonisateur jusqu'à la colonie sud-américaine, mais aussi du parcours de la langue portugaise d'un continent vers l'autre.

36. (23) : Il y a beaucoup de diversité (à Montréal) avec les nombreux Italiens ou les Grecs.

37. (24) : Quand nous sommes arrivés ici, il n'y avait rien.

38. (25) : Il y avait déjà des postes (de travail) trouvé par la famille (au Canada). 
Ce passage de la langue portugaise du continent européen jusqu'au-delà de l'océan Atlantique, peut être analysé comme un «voyage» linguistique qui est fait en passant par les archipels de Madère et des Açores.

Si selon les traits phonétiques, une variation régionale et une différentiation se maintiennent entre Nord et îles, du point de vue des traits structurels cette variation semble vouloir converger vers des normes de pratiques communes. Les institutions et les médias auraient alors un rôle important.

Au Portugal, la variante du sud serait celle qui se rapproche le plus du PB et du PI. La variante méridionale, force d'expansion se serait imposée au passage d'abord dans les îles et ensuite au Brésil (Cintra, 1971). Ce voyage du portugais se rapproche de celui de l'espagnol et de son implantation en Amérique latine. Dans les deux cas, ce sont les langues du nord de la péninsule Ibérique, le castillan et le galicien-portugais, qui ont initié ce voyage, tout d'abord vers le sud de la péninsule, puis en passant par les îles pour finalement se répandre dans le nouveau monde colonial. En ce qui concerne l'espagnol et ses variantes, on constate une proximité majeure entre les variantes du sud de l'Espagne (Andalousie et îles Canaries) et les variantes de l'espagnol d'Amérique latine. Pour le portugais et ses variantes aussi, nous pouvons souligner cette proximité entre les variantes du sud du pays (Alentejo et Algarve) et celles des archipels avec le portugais parlé au Brésil (PB).

Comme nous l'avons constaté, certains éléments structurels sont communs à la variante méridionale du PE et au PB : la construction des verbes d'aspect avec le mode gérondif, l'usage et la concordance de l'expression a gente comme sujet et les constructions impersonnelles du verbe auxiliaire ter existentiel. Cependant, ces constructions, même si qualifiées de non normatives en $\mathrm{PE}$, ont été étudiées dans une perspective géolinguistique en tant que «variations dialectales du territoire national portugais » (Carrilho \& Pereira, 2011).

Il est intéressant d'observer le statut de référence qu'a acquis le PB au fil des ans et son influence sur les autres portugais. Il est important, par exemple, de rappeler l'importance qu'a eu le PB dans la conception de l'accord orthographique de la langue portugaise, «Acordo ortográfico» $\left(\mathrm{AO} 90^{39}\right)$. Dans notre image de «voyage» du portugais, nous voyons une simplification et une convergence vers ce $\mathrm{PB}$, variante dominante dans les médias mais aussi choisie dans l'enseignement (Oliveira, 2013).

39. AO90 - Acordo Ortográfico da língua portuguesa de 1990, Accord orthographique de la langue portugaise de 1990 (Oliveira, 2013). 
Pour conclure, il a été intéressant d'observer le portugais utilisé et qui circule grâce aux institutions, comme les écoles ou les médias. La position centrale et de pouvoir de la norme, soit du PE soit du PB, est importante dans les pratiques à l'oral, mais aussi à l'écrit (écriture, lecture, sous-titrage des films), et trouve un croisement dans la construction discursive pour légitimer la «bonne» référence.

PE et PB s'inscrivent dans une lutte pluricentrique (Clyne, 1992; Muhr, 2012), qui permet aux variantes dialectales tout autour de survivre en diminuant la distance géographique entre les espaces. À Montréal, au sein de la comunidade, ces traits de variation dialectale survivent dans le temps et s'inscrivent comme des marqueurs d'identité de certains locuteurs et locutrices au sein de la portugaisité montréalaise.

\section{RÉFÉRENCES BIBLIOGRAPHIQUES}

Almeida Erika, 2014, «La citoyenneté au-delà des frontières nationales : la politique étrangère brésilienne et les Brésiliens au Québec », RITA, $\mathrm{n}^{\circ} 7$.

Almeida Moura Manuel \& Soares Imitério, 2003, Pionniers. L'avant-garde de L'immigration portugaise, Canada 1953, Montréal, Direcção Geral dos Assuntos Consulares e Comunidades Portuguesas.

Anderson Benedict, 1992, «The New World Disorder», New Left Review, n 193, p. 4-11.

ANDRADe Ernesto Pardal D’, 1993, «Algumas particularidades do português falado no Funchal», dans Actas do IX Encontro da Associação Portuguesa de Linguística, Coimbra/Lisbonne, Éd. Colibri, p. 17-29.

BAZENGa Aline, 2012, «Construcciones existenciales: variación sintáctica y variedades del portugués », communication présentée à la $X^{\mathrm{e}}$ Conférence internationale de linguistique générale (CILG), Saragosse, Universidad de Zaragoza.

Benítez Fernández Montserrat, 2017, «Variation linguistique dans le parler arabe d'Oujda : Nord-Est marocain vs. Communauté marocaine dans la diaspora», Dialectologia, $\mathrm{n}^{\circ} 18$, p. 1-18.

CAnut Cécile, 2000, «Subjectivité, imaginaires et fantasmes des langues : la mise en discours épilinguistique», Langage et Société, vol. 93, nº 3, p. 71-97.

CARRILho Ernestina \& Pereira Sandra, 2011, «Sobre a distribuição geográfica de construções sintácticas não-padrão em português europeu», dans Actas do XXVI Encontro da Associação Portuguesa de Linguística (APL), Lisbonne, p. 125-139.

CASTro Ivo, 2006, Introdução a história do português. Geografia da língua. Português Antigo, Lisbonne, Éd. Colibrí.

CAubet Dominique, 2001, «L'arabe dialectal en France», Arabofrancophonie. Les Cahiers de la francophonie, $\mathrm{n}^{\circ}$ 10, Paris, L'Harmattan, p. 199-212. 
Chambers Jack J.K., Trudgill Peter \& Schilling-Estes Natalie (éds), 2002, The Handbook of Language Variation, Oxford, Blackwell.

Cintra Luís F. Lindley, 1971, «Nova Proposta de classificação dos dialectos galego-portugueses », Boletim de Filologia, n 22, p. 81-116.

Cintra Luís F. Lindley, 1990, «Os Dialectos da Ilha da Madeira no Quadro Geral dos Dialectos Galego-Portugueses», dans J. E. Franco (éd.), II Congresso da Cultura Madeirense, Porto, Campo das Letras, p. 95-104.

Clyne Michael, 1992, Pluricentric Languages. Differing Norms in Different Nations, Berlin et New York, Mouton de Gruyter.

DABÈnE Louise, 1994, Repères sociolinguistiques pour l'enseignement des langues, Paris, Hachette.

Da Rosa Pereira Victor \& Teixeira Carlos, 2000, The Portuguese in Canada: Diasporic Challenges and Adjustment, Toronto, University of Toronto Press.

Deprez Christine, 2005, «Langues et migrations : dynamiques en cours », La linguistique, vol. 41, $\mathrm{n}^{\circ}$ 2, p. 9-22.

Deprez Christine, 2013, «Le Portugais », dans G. Kremnitz, Histoire sociale des langues de France, Rennes, Presses universitaires de Rennes, p. 799-804.

Donabédian Anaïd, 2000, «Langues de diaspora, langues en danger : le cas de l'arménien occidental», Mémoires de la Société de linguistique de Paris, nouvelle série, t. VIII [Les Langues en danger], p. 137-156.

ERICKSON Frederick, 1984, «What Makes School Ethnography 'Ethnographic'?», Anthropology and Education Quarterly, n 15, p. 51-66.

EuséBIo Joaquim, 2001, Falando Português em Montréal, Montréal, Quebec World.

Fishman Joshua A., 1971, Sociolinguistics: A Brief Introduction, Rowley, Newbury House.

FITch Roxana, 2013, «Comparison of Colloquial Terms in Two Mexican Spanish Dictionaries: An Analysis Based on Examples of Use. "Gacho" and "Ultimadamente" », Procedia - Social and Behavioural Sciences, n 95, p. 308-316.

GonçAlves Anabela, 1996, «Aspectos da sintaxe dos verbos auxiliares do Português Europeu », dans A. Gonçalves, M. Colaço, M. Miguel \& T. Móia (éds), Quatro estudos em Sintaxe do Português. Uma abordagem segundo a Teoria dos Princípios e Parâmetros, Lisbonne, Éd. Colibri.

Hymes Dell Hymes, 1996, Ethnography, Linguistics, Narrative Inequality: Toward an Understanding of Voice, Londres, Taylor \& Francis.

Leite Yvonne, Callou Dinah \& Moraes João, 2003, «Processos de mudança no português do Brasil: variáveis sociais », dans I. Castro \& I. Duarte (éds), Razões e emoção: miscelânea de estudos em homenagem a Maria Helena Mira Mateus, Lisbonne, Imprensa nacional-Casa da moeda, p. 87-114.

Lово Maria, 2008, «Variação morfo-sintáctica em dialectos do português europeu: o gerúndio flexionado », Diacrítica, vol. 22, n 1, Braga, Universidade do Minho, p. 25-55.

MACKey William Francis, 1989, «Politique et aménagement linguistique : une discipline ou deux?», Langues et linguistique, $\mathrm{n}^{\circ}$ 15, p. 123-163. 
Maia de Azevedo Clarinda, 1977, «Os falares fronteiriços do concelho do Sabugal e da vizinha regiao de Xalma e Alamedilla», Suplemento IV da Revista Portuguesa de Filologia, Coimbra.

Martins Ana Maria, 2003, «Construções com se: mudança e variação no Português Europeu», dans I. Castro \& I. Duarte (éds), Razões e emoção: miscelânea de estudos em homenagem a Maria Helena Mira Mateus, Lisbonne, Imprensa nacional-Casa da moeda.

Mateus Maria Helena Maia, 2002, «Variação e variedades: o caso do Português », dans S. Grosse \& A. Schönberger, Ex Oriente lux. Festschrift für Eberhard Gärtner zu seinem 60 Geburtstag, Francfort-sur-le-Main, Valentia, p. 287-296.

Mateus Maria Helena Maia, 2005, «A mudança da língua no tempo e no espaço», dans M.H.M. Mateus \& F. Bacelar, A Língua Portuguesa em Mudança, Lisbonne, Editorial Caminho, p. 13-30.

Matzenauer Carmen Lúcia Barreto, 1999, «Aquisição da fonologia e aplicações teóricas: um estudo sobre as soantes palatais », dans R. R. Lampretch (éd.), Aquisição da Linguagem: questões e análises, Porto Alegre, EdiPUCRS.

Mazzoli Maria, 2005, Portuguese migrants in Andorra, Report of the fieldwork conducted in Andorra July-November 2015 for the MIME project, University of Milano-Bicocca.

Montrul Silvina, 2010, «How Similar Are Adult Second Language Learners and Spanish Heritage Speakers? Spanish Clitics and Word Order», Applied Psycholinguistics, $\mathrm{n}^{\circ}$ 31, p. 167-207.

Montrul Silvina, 2012, «Is the Heritage Language Like a Second Language?», EUROSLA Yearbook, vol. 12, p. 1-29.

MuHr Rudolf, 2012, «Linguistic Dominance and Non-Dominance in Pluricentric Languages: A Typology», dans R. Muhr (éd.), Non-Dominante Varieties of Pluricentric Languages. Getting the Pictures. In Memory of Michael Clyne, Vienne, Peter Lang Verlag.

Nobre Correia José Manuel, 1997, «Les médias portugais. De la dictature à la démocratie», MédiasPouvoirs, $\mathrm{n}^{\circ}$ 1, p. 37-47.

Oliveira Gilvan M., 2013, «Política linguística e internacionalização: A língua portuguesa no mundo globalizado do século XXI», Trabalhos em Linguística Aplicada, vol. 52, n 2, p. 409-433.

Pereira Sílvia Afonso, 2014, «A sintaxe na classificação dos dialetos portugueses», dans Textos selecionados: XXIX Encontro nacional da Associação Portuguesa de Linguística, Porto, APL, p. 445-464.

PolášEK Metoděj, 2004, O falar da Ilha da Graciosa - Açores, Magisterská diplomová práce (thèse de master), Brno, FFMU.

Rebelo Helena, 2005, «A caminho do Brasil: situação actual da língua portuguesa na Ilha da Madeira», Revista Xarabanda, n 16, p. 64-73.

Rotнмал Jason, 2009, «Understanding the Nature of Early Bilingualism: Romance Languages As Heritage Languages », Special Issue of International Journal of Bilingualism, vol. 13, n 2, p. 155-163.

Scaglione Stefania, 2000, Attrition. Mutamenti sociolinguistici nel lucchese di San Francisco, Milan, Franco Angeli. 
ScETTI Fabio, 2016, Évolution de la langue portugaise dans sa dynamique de transmission au sein de la «communauté portugaise» de Montréal, thèse de doctorat, Université Paris Descartes, Paris.

Segura Luísa, 2006, «Dialectos açorianos. Contributos para a sua classificação», dans M. C. Bernardo \& H. Mateus Montenegro (éds), Encontro de estudos dialectológicos - Actas (Ponta Delgada, 2003), Ponta Delgada, Instituto Cultural de Ponta Delgada, p. 325-344.

Segura Luísa, 2013a, «Geografia da língua portuguesa», dans Gramática do Português, vol. I, Lisbonne, Fundação Calouste Gulbenkian.

SEgura Luísa, 2013b, «Variedades dialetais do português Europeu », dans Gramática do Português, vol. I, Lisbonne, Fundação Calouste Gulbenkian.

Segura Luísa \& Saramago João, 1999, «Açores e Madeira: autonomia e coesão dialectais », dans I. H. Faria (éd.), Lindley Cintra: Homenagem ao Homem, ao Mestre e ao Cidadão, Lisbonne, Cosmos \& Faculdade de Letras da Universidade de Lisboa.

Segura Luísa \& Saramago João, 2001, «Variedades dialectais portuguesas », dans Caminhos do Português: Exposição comemorativa do ano europeu das línguas (Catálogo).

Silva-Corvalán Carmen, 1994, «The Gradual Loss of Mood Distinctions in Los Angeles Spanish», Language Variation and Change, ${ }^{\circ}$ 6, p. 255-272.

Teyssier Paul \& Maia de Azevedo Clarinda, 1988, «Historia do Galego-Português. Estado lingüístico da Galiza e do Noroeste de Portugal desde o século XIII ao século XVI (Com refêrencia à situaçâo do galego moderno), Instituto Nacional de Investigaçâo Científica (Lingüística 9), Coimbra, 1986 », Cahiers de linguistique hispanique médiévale, $\mathrm{n}^{\circ} 13, \mathrm{p} .62-73$.

Trudgill Peter, 1986, Dialects in Contact, Oxford, Blackwell.

Tzen Ming Daniel, 2000, Nomination des lieux en France par les Chinois de France, en mandarin, thèse de doctorat, Université Paris Descartes.

Vicente Ángeles, 2007, «Two Cases of Moroccan Arabic in the Diaspora», dans C. Miller, E. Al-Wer, D. Caubet \& J. C. E. Watson (éds), Arabic in the City. Issues in Dialect Contact and Language Variation, Londres, Routledge, p. 123-143.

Wetzels W. Leo, 2000, «Consoantes palatais como geminadas fonológicas no Português Brasileiro », Revista de Estudos Linguísticos, vol. 9, n 2, p. 5-15.

Zentella Ana Celia, 1997, Growing Up Bilingual: Puerto Rican Children in New York, New York, Wiley and Sons.

Zheng Li-Hua, 1995, Les Chinois de Paris et leurs jeux de face, Paris, L'Harmattan, coll. «Logiques sociales».

Statistiques Canada, 2003, Census of Canada (2001) - Profile of Ethnic Category, Mother Tongue and Home Language, Ottawa, Industry Canada.

Statistiques Canada, 2009, Census of Canada (2006) - Citizenship, Immigration, Birthplace, Generation Status, Ethnic Origin, Visible Minorities, and Aboriginal Peoples, 97-557-XIE, Ottawa, Industry Canada. 